\title{
Multiagent Simulation of the Hepatitis B Epidemic Process
}

\author{
Tetyana Chumachenko*1, Dmytro Chumachenko² and Olexandr Sokolov ${ }^{2}$ \\ ${ }^{1}$ Epidemiology Department, Kharkiv National Medical University, Kharkiv, Ukraine; ${ }^{2}$ National Aerospace University "Kharkiv Aviation \\ Institute", Kharkiv, Ukraine
}

\section{Objective}

To develop multiagent model of hepatitis B (HBV) infection spreading.

\section{Introduction}

The standard approaches to simulation include solving of differential equation systems. Such approach is good for obtaining general picture of epidemics $(1,2)$. When the detailed analysis of epidemics reasons is needed such model becomes insufficient. To overcome the limitations of standard approaches a new one has been offered. The multiagent approach has been offered to be used for representation of the society. Methods of event-driven programming give essential benefits of the processing time of the events (3).

\section{Methods}

For model development $\mathrm{C} \#$ computing language has been used. We have used demographical data, the incidence rate of HBV infection of all population and different population groups (age, professional and other groups), coverage of hepatitis B vaccination, the proportion of HBV carriers in population, the prevalence rate of chronic HBV infection, percent of dominated transmission routes and factors and other rates in Kharkiv region. All parameters, expressed in the model were estimated using sero-surveys data and data of epidemiological surveillance of Kharkiv region sanitary-epidemiological station. Also the theoretical knowledge about HBV infection has been used. 26 conditions have been derived from the problem domain. The transition from one condition to another depends on stochastic value and time of the event change. All events are organized in priority queue which results in high rate of computation performance. The dependence on time and random value determines automata theory conceptions.

\section{Results}

The prototype of software system, which includes a subsystem of the multiagent simulation and specialized statistical and mathematical sub-system which can process the simulation results and perform a conditional optimization of the selected objective functions (morbidity, the effectiveness of specific preventive and control activities and their price, measure of reducing the socio-economic impact of HBV infection, etc.) have been developed. Screen form is presented in Figure 1.

\section{Conclusions}

The multiagent simulation model of the HBV infection epidemic situation development, based on data obtained in Kharkiv (Ukraine) has been created.
The simulation results allow us to:

1. predict the dynamics of the epidemic process in time in a particular area, taking into account specific epidemic situation;

2. test the effectiveness of various preventive measures (sterilization of instruments, coverage of hepatitis B vaccination of certain groups of people, etc.).

Using the present model in public health system suggests improvment of the epidemiological diagnostics of HBV infection and of the quality of management decisions about epidemiological surveillance. The evolution of multiagent simulation in epidemiology will broaden the possibilities of epidemiological surveillance and control.

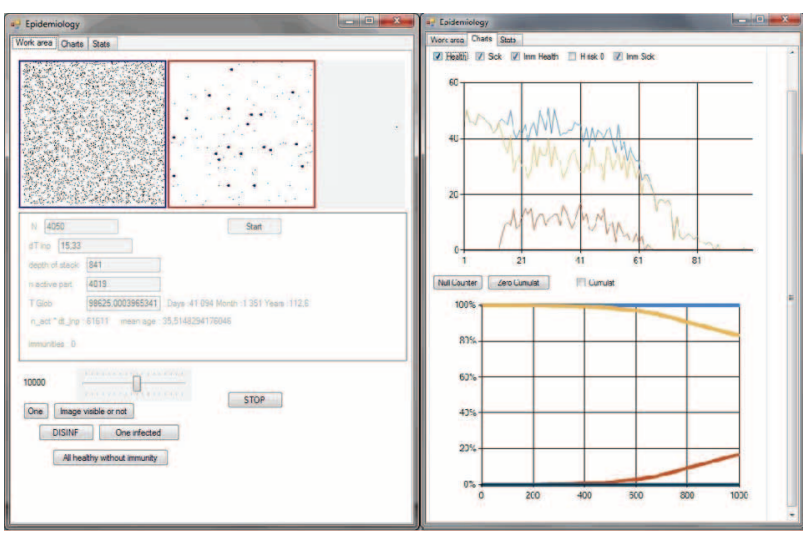

Fig. 1. The main panel of simulation management and graphic visualization.

\section{Keywords}

Multiagent modeling; Event-driven programming; Predictive disease modeling; Epidemiological surveillance

\section{References}

1. Shoujun Zhao, Zhiyi Xu, Ying Lu.: Mathematical model of hepatitis B virus transmission and its application for vaccination strategy in China. International Journal of Epidemiology. 2000;29:744-52.

2. O'Leary, C., Hong, Z., Zhang, F., Dawood, M., Smart, G., Kaita, K., $\mathrm{Wu}, \mathrm{J}$ : : A mathematical model to study the effect of hepatitis B virus vaccine and antivirus treatment among the Canadian Inuit population.Eur J Clin Microbiol Infect Dis. 2010 Jan;29(1):63-72.

3. Hethcote, H.W.: The mathematics of infectious diseases. SIAM Rev. 2000; 42(4): 599-653.

*Tetyana Chumachenko

E-mail: tatalchum@gmail.com 